\title{
A NOTE ON THE THOM ISOMORPHISM
}

\author{
MARK MAHOWALD ${ }^{1}$ AND NIGEL RAY \\ ABSTRACT. The generalized homology version of the Thom isomorphism theorem is \\ exploited to give easy proofs of several recent theorems.
}

Let $\Omega$ be an $H$-space with homotopy inverse $x \rightarrow x^{-1}$, and let $f: \Omega \rightarrow B F$ classify a stable spherical fibration over $\Omega$. The following result is proved in [2].

THEOREM 1. If $f$ is an $H$-map, then the Thom spectrum $T(f)$ is a ring spectrum, and there is a homotopy equivalence $\alpha: T(f) \wedge \Omega_{+} \cong T(f) \wedge T(f)$ (where + signifies a disjoint base point).

For many applications, it is improtant that $\alpha$ is given by the Thomification of an explicit map $g: \Omega \times \Omega \rightarrow \Omega \times \Omega$, where $g(x, y)=\left(x y^{-1}, y\right)$. For details, see [2].

Our purpose here is to note that Theorem 1 follows from a strong form of the Thom isomorphism theorem in generalized homology. Specifically, we assume given a stable spherical fibration $\nu: X \rightarrow B F$ over a locally finite CW complex $X$, and a ring spectrum $E$ orienting $\nu$; i.e. a Thom class $U: T(\nu) \rightarrow E$ whose restriction to a fibre $S^{0} \hookrightarrow T(\nu)$ is the unit of $E$. Then we have

THEOREM 2. There is a homotopy equivalence $\alpha(U): E \wedge T(\nu) \rightarrow E \wedge X_{+}$which on homotopy groups induces the traditional Thom isomorphism

$$
\phi_{U}: E_{*}(T(\nu))=\pi_{*}(E \wedge T(\nu)) \underset{\alpha(U)}{\stackrel{\simeq}{\rightarrow}} \pi_{*}\left(E \wedge X_{+}\right)=E_{*}\left(X_{+}\right) .
$$

Proof. First suppose that $X$ has finite dimension, so that $\nu$ lifts to $\nu_{n}: X \rightarrow B F_{n}$ for suitably large $n$. Let $p_{n}: S\left(\nu_{n}\right) \rightarrow X$ be the asociated $n$-sphere fibration, so that $T\left(\nu_{n}\right)=X \cup_{p_{n}} \operatorname{CS}\left(\nu_{n}\right)$.

Now write $\Delta: T\left(\nu_{n}\right) \rightarrow T\left(\nu_{n}\right) \wedge X_{+}$for the diagonal

$$
x \mapsto \begin{cases}\left(x, p_{n}(x)\right), & x \neq \infty, \\ \infty, & x \neq \infty,\end{cases}
$$

and consider the composite

$$
\begin{aligned}
\alpha(U): E \wedge T\left(\nu_{n}\right) \underset{\mathrm{id} \wedge \Delta}{\rightarrow} E & \wedge T\left(\nu_{n}\right) \wedge X_{+} \\
& \quad \underset{\operatorname{id} \wedge}{\vec{U} \wedge \text { id }} E \wedge \Sigma^{n+1} E \wedge X_{+} \underset{\mu \wedge \text { id }}{\rightarrow} E \wedge \Sigma^{n+1} X_{+}
\end{aligned}
$$

wher $\mu$ is the product in $E$.

Received by the editors August 4, 1980.

1980 Mathematics Subject Classification. Primary 55N20, 55P10.

1 The first named author is supported in part by NSF Grant MCS 76-07051. 
On homotopy groups this map induces a homomorphism $\phi_{U}: E_{*+n+1}\left(T\left(\nu_{n}\right)\right) \rightarrow$ $E_{*}\left(X_{+}\right)$which, by very construction, is $\cap U$. Thus $\phi_{U}$ is the usual Thom isomorphism, whence $\alpha(U)$ is an equivalence.

To complete the proof for all $X$, let $n \rightarrow \infty$.

COROLlary 3. Theorem 1 is true.

Proof. Let $X=\Omega$; choose $E=T(\nu)$ and $U$ as the identity. Then $\alpha$ (id): $T(\nu) \wedge$ $T(\nu) \underset{\sim}{\longrightarrow} T(\nu) \wedge \Omega_{+}$is just the Thomification of $g^{\prime}: \Omega \times \Omega \rightarrow \Omega \times \Omega$ given by $g^{\prime}(x, y)$ $=(x y, y)$. Thus the inverse of $\alpha(\mathrm{id})$ is the Thomification of $g^{\prime-1}$, and $g^{\prime-1}$ is clearly g.

Corollary 4. Suppose $\nu: X \rightarrow B O$ lifts to the $(i-1)$-connected cover $B O\langle i\rangle$. Let $M O\langle i\rangle$ be the corresponding Thom spectrum. Then there is an equivalence $\beta$ : $M O\langle i\rangle \wedge T(\nu) \rightarrow M O\langle i\rangle \wedge X_{+} \cdot$

Applying this, we deduce a result of [1].

Corollary 5. Let $N(i)$ be such that $N(i) \xi$ is trivial over $R P^{i}$, where $\xi$ is the Hopf line bundle. Then there is an equivalence $M O\langle i\rangle \wedge R P_{N(i)+j}^{\infty} \rightarrow M O\langle i\rangle \wedge \Sigma^{N(i)} R P_{j}^{\infty}$ for all $j$ (where $\left.R P_{n}^{m}=R P^{m} / R P^{n-1}\right)$.

Proof. Consider the composite

$$
\begin{aligned}
M O\langle i\rangle \wedge R P_{N(i)+j}^{\infty} & \rightarrow M O\langle i\rangle \wedge R P_{N(i)}^{\infty} \wedge R P_{j}^{\infty} \\
& \stackrel{\beta \wedge \text { id }}{\longrightarrow} M O\langle i\rangle \wedge \Sigma^{N(i)} R P_{+}^{\infty} \wedge R P_{j}^{\infty} \rightarrow M O\langle i\rangle \wedge \Sigma^{n(i)} R P_{j}^{\infty},
\end{aligned}
$$

where the first map is induced by the diagonal $T(N(i) \xi \oplus j \xi) \rightarrow T(N(i) \xi) \wedge T(j \xi)$, and the third by the projection $R P_{+}^{\infty} \rightarrow S^{0}$.

A simple homology calculation shows the composite to be an equivalence.

\section{REFERENCES}

1. D. M. Davis, S. Gitler, W. Iberkleid and M. Mahowald, Orientability of bundles with respect to certain spectra, Bol. Soc. Mat. Mexicana (to appear).

2. M. Mahowald, Ring spectra which are Thom complexes, Duke Math. J. 46 (1979), 549-559.

Department of Mathematics, NorhWESTERn University, Evanston, IlLnNOIS 60201

Department of Mathematics, The University of Manchester, Manchester, England M13 9PL 